Revue

Revue de l'histoire des religions

de Ihistoire des religions

3 | 2019

Varia

Corinne BonNET, Gabriella PIRONTI (dir.), Les dieux d'Homère. Polythéisme et poésie en Grèce ancienne

Liège, Presses Universitaires de Liège (« Kernos », supplément 31), 2017

\title{
Magali Année
}

\section{(2) OpenEdition}

Journals

Édition électronique

URL : https://journals.openedition.org/rhr/9953

DOI : 10.4000/rhr.9953

ISSN : 2105-2573

Éditeur

Armand Colin

Édition imprimée

Date de publication : 1 septembre 2019

Pagination : 597-600

ISBN : 978-2-200-93260-2

ISSN : 0035-1423

Référence électronique

Magali Année, « Corinne Bonnet, Gabriella Pirontı (dir.), Les dieux d'Homère. Polythéisme et poésie en Grèce ancienne », Revue de l'histoire des religions [En ligne], 3 | 2019, mis en ligne le 01 janvier 2021 consulté le 08 janvier 2022. URL : http://journals.openedition.org/rhr/9953 ; DOI : https://doi.org/ 10.4000/rhr.9953

Ce document a été généré automatiquement le 8 janvier 2022

Tous droits réservés 


\section{Corinne BONNET, Gabriella PironTi (dir.), Les dieux d'Homère. Polythéisme et poésie en Grèce ancienne}

Liège, Presses Universitaires de Liège (« Kernos », supplément 31), 2017

Magali Année

\section{RÉFÉRENCE}

Corinne BONNET, Gabriella PIRONTI (dir.), Les dieux d'Homère. Polythéisme et poésie en Grèce ancienne, Liège, Presses Universitaires de Liège (« Kernos », supplément 31), 2017, 257 p., $24 \mathrm{~cm}, 25 €$, ISBN 978-2-87562-130-6.

1 Alors que ces dernières décennies ont vu croître le nombre d'études consacrées aux polythéismes anciens, que face aux dérives fanatiques, actuelles et passées, des monothéismes occidentaux, l'urgence s'est peut-être fait sentir de plus en plus forte de (re)prendre au sérieux les conceptions polythéistes du monde dans leur propre diversité et dans leur différence radicale mais non pour autant conflictuelle à l'égard de notre « moule monothéiste » (p. 12, voir aussi les stimulantes p. 177-180 du chap. viI de David Bouvier), "le véritable travail d'équipe " que constitue l'ouvrage dirigé par Corinne Bonnet et Gabriella Pironti comble, avec la simplicité d'un résultat magistral, un manque évident. Entre l'« éloge» historico-anthropologique du polythéisme (cf. Maurizio Bettini, Elogio del politeismo. Quello che possiamo imparare oggi dalle religioni antiche, Bologne, Il Mulino, 2014 [Belles Lettres, 2016]) et le constat philosophique, voire spirituellement assumé, qu'll y a des dieux (titre de l'ouvrage de Frédérique Ildefonse, PUF, 2012), il remplit en effet à merveille le double objectif qu'il s'est fixé : celui d'« inaugurer une lecture [authentiquement] "polythéiste" des récits homériques" (p.13) et celui de raviver en nous, outre le plaisir reconnaissant d'une «lecture intégrale des poèmes homériques » (p. 10), « l'expérience exaltante dans l'immersion partagée [de ce qu'ils nous disent de] l'univers culturel des Grecs » (p. 17). 
2 Mieux que ne le fait encore l'introduction-manifeste des deux directrices (p. 7-17), c'est peut-être la justesse du titre elle-même, sous l'éclairage rétroactif et en quelque sorte annulaire que lui apporte en substance le tout dernier chapitre de Miguel Herrero de Jáuregui consacré à la notion de "salut" (chap. Ix, p. 203-228), qui rend le plus fidèlement compte de l'entreprise collective : d'un côté, montrer que Les dieux d'Homère sont à la fois les dieux de l'imaginaire religieux de tous les Grecs et en même temps les dieux spécifiques d'Homère, agissant de conserve au nom d'un ordre aussi bien cosmique que narratif; de l'autre, laisser à comprendre que si les « méandres de ce polythéisme " à la fois général et particulier (p.11) sont aussi indéfectiblement intriqués avec la trame narrative des poèmes c'est qu'ils relèvent moins d'une "religion » (au sens moderne du terme) que d'une tradition poético-religieuse. La "religion " grecque et les pratiques cultuelles qui la définissent, à l'époque archaïque, existent dans et par la poésie qui les dit pour la simple raison que la langue poétique était alors le seul véhicule du savoir et, plus encore que les figurations iconographiques, le support-roi, et pour ainsi dire vivant, "d'une pensée qui appréhende le monde, qui l'explore et s'efforce de l'expliquer, dans sa complexité relationnelle» (p.13). Ce qui nous donne peut-être mieux à comprendre pour lui-même le fameux dit d'Hérodote (Histoires II, 53) et explique qu'il revienne comme un leitmotiv de chapitre en chapitre (p. 8 ; chap. I, p. 22 ; chap. II, p. 43 ; chap. VI, p. 135 ; chap. VII, p. 157) : ce sont de fait inévitablement Homère et Hésiode qui, pour les Grecs, ont circonscrit l'aspect des dieux et leur sphère de compétences.

Premier d'une série d'ateliers sur les dieux d'Homère, initiée à Rome en 2015, le volume rassemble les versions françaises de textes d'abord publiés en italien chez Carocci. Elles sont suivies d'une bibliographie substantielle d'une dizaine de pages, d'un index général des noms et des notions (qu'on aurait aimé plus fourni et plus précis : à titre d'exemple, l'entrée anthropomorphisme ne référence pas les pages où seul l'adjectif est employé) et d'un index des principaux passages cités. Formant une somme de neuf chapitres, ces textes s'organisent trois à trois en trois parties cohérentes, que l'on pourrait subsumer sous le triptyque "représentation / inter-communication / réflexivité ». La première (Raconter les puissances divines, p.21-83) s'attache aux stratégies narratives de représentation du divin; la deuxième (Entre l'olympe et la terre, p. 87-150) se concentre sur le processus de communication complexe entre les dieux eux-mêmes autant qu'entre les dieux et les hommes; la troisième (De la guerre au salut, p. 153-228) montre à quel point la guerre et la mort qu'elle implique permettaient de penser intrinsèquement le monde du divin et combien celui-ci en retour permettait de réfléchir sur le problème humain de la guerre et de la mort. Mais par-delà cette organisation, la plus belle réussite de l'ouvrage est de constituer un ensemble à l'image même de son objet, dont le maître-mot n'est autre que fluidité. Comme le " câble d'or " dont parle C. Bonnet (p. 95-96), qui relie tous les dieux ensemble et à Zeus (Il. 8, 19-27) pour en faire un véritable "organisme vivant et relationnel ", "malléable mais structuré ", et en perpétuelle reconfiguration (p.103), les neuf chapitres s'articulent en une même chaîne flexible et souple où se tissent sans cesse des interactions mouvantes et multiples entre les diverses analyses et approches qui sont celles de leurs auteurs respectifs - historiens ou anthropologues, experts des religions anciennes ou spécialistes de littérature grecque (voir, à ce titre, la revendication « symphonique » de la n. 9, p. 155 du chap. viI de Pascal Payen). 
4 Ainsi, par une attention méticuleuse portée à la trame narrative de l'Iliade, le chapitre remarquable de G. Pironti (chap. III, p. 63-83) ne se contente pas de nous révéler le dédale de la volonté de Zeus (la Dios boulē), résultat de la «dynamique conflictuelle inhérente à la relation privilégiée entre le roi des dieux et son épouse »-ladite teleia, comme le rappelle C. Bonnet (chap. IV, n. 15, p. 95), qui permet l'« accomplissement » de sa souveraineté. En dévoilant dans toute sa complexité la dimension poético-religieuse du chant épique où, à certains nœuds d'importance théologique et narrative, Dios boulē et inspiration aédique coïncident en une adéquation parfaite (comme lors de la Dios apatē du chant 14, où Zeus est aussi trompé que séduit par Héra), la démonstration entre directement en résonance avec les développements des chap. IV et IX, qui se renvoient l'un à l'autre, ainsi qu'avec cette interrogation de D. Bouvier (p. 198): «[apparente] incohérence de Zeus ou discours double d'un dieu qui connaît à l'avance les réactions de ses interlocuteurs?». D'un côté (chap. Iv), la décision de Zeus s'avère sans cesse soumise à négociation dans le cadre d'assemblées divines vues à travers le miroir de l'agora civique des hommes, tantôt pertuis tantôt trou noir mais seul moyen d'entrevoir l'insaisissable eukosmia du monde divin (p. 105 et 112) ; de l'autre (chap. Ix), on la voit finalement toujours se confondre avec le consensus de tous les dieux et finir par abolir la distinction numérique theos/theoi (p. 216-217). Nous sommes alors renvoyés à l'insuffisance des prières humaines (chap. vi de Vinciane Pirenne-Delforge); à cette oscillation des dieux, que nous laissent voir la plupart des chapitres, entre hostilité, bienveillance et indifférence à l'égard des hommes; et encore - pace Xénophane, cité presque autant de fois qu'Hérodote - à l'altérité radicale de leurs manifestations synesthésiques (chap.II d'Adeline Grand-Clément) et de leur anthropomorphisme «diamorphique» (chap.I, p.26) qui mériterait, nous dit M. Bettini (p.39), d'être repensé plutôt comme un " "théomorphisme" des humains ".

5 La place manque pour s'arrêter sur les qualités de chacun des chapitres, mais qu'il suffise de reconnaître à tous la réussite d'avoir montré, sans jamais la figer, la pensée grecque archaïque au travail, une pensée unitaire et globalisante du monde où tout se tient entrelacé dans une mouvance protéiforme infinie: les dieux les uns avec les autres, dont les modalités réactives d'intervention (p.129-130) se recouvrent régulièrement (comme la messagère Iris se mettant à faire le héraut Hermès, chap. $\mathrm{v}$ de Carmine Pisano) ; les dieux et l'ordre cosmique ; les dieux et les hommes ; les frontières mêmes qui séparent leurs deux mondes apparemment radicalement différents, puisqu'il arrive à Protée lui-même de douter de la nature divine ou humaine de Ménélas (Od. 4, 462; p. 212) ; le discours «théologique » et la guerre des hommes; ou même encore la flexibilité fondamentale du polythéisme et la fluidité sémiologique et métamorphique inhérente à la langue grecque elle-même (cf. chap. viII, p. 187-188 et chap. IX, p. 224).

6 Ainsi des dieux d'Homère, au bout du compte, a-t-on bel et bien appris « [à] parler et [à] penser autrement ». Car ils «ne sont pas quelque part entre littérature, mythologie et religion. Ils sont dans la pensée grecque le moyen de croire et de comprendre des réalités que les Grecs anciens avaient choisi de formuler ainsi » (D. Bouvier, p. 188 puis 199). On finira également convaincu que langue, culture et religion (pour reprendre une triade toute benvenistienne) s'interpénètrent et se confondent décidément pour ne former qu'une seule et même globalité. 


\section{AUTEURS}

MAGALI ANNÉE

UMR 8163 - Savoirs, Textes, Langage (Lille). 